\title{
Undergraduate Optoelectronics Laboratories
}

\author{
Susan M. Lord \\ Bucknell University
}

\begin{abstract}
This Instrumentation and Laboratory Improvement project focuses on providing undergraduates with experience in optoelectronics, an important multidisciplinary technology. An Optoelectronics Laboratory facility has been established at Bucknell University. This enabled the development of laboratory experiments for first-year students and for juniors, seniors, and masters students in an elective course.
\end{abstract}

A laboratory experiment was performed by 215 first year engineering and other interested students in the fall of 1996. This exercise included five inexpensive and transportable modules which provide a hands-on introduction to optoelectronics.

In the spring of 1996, students in "Optoelectronic Materials and Devices" performed five new laboratory experiments. These experiments included determining the lattice parameters of semiconductors from X-ray diffraction data, comparing white light sources, gas and semiconductor lasers, and light emitting diodes (LEDs), characterizing materials for detectors and comparing photodetector device structures. The final two experiments focused on fiber optics and were developed by a student as her senior design project. Students coupled light from a HeNe laser into an optical fiber in one lab. In "Creating Optical Communication Links", students investigated digital and analog modulation using plastic fiber, glass fiber, and free space. Finally, students multiplexed two stereo signals: one using an external modulator and one using a directly modulated LED.

\section{INTRODUCTION}

Optoelectronics involves using electrons (electricity) and photons (light or optical energy) to perform useful functions. Optoelectronics is an area of growing importance in engineering and in the consumer market. A multidisciplinary endeavor combining elements of physics, electrical engineering, and materials science, optoelectronics is also a good forum to introduce students to the need to bring together information from many different technical fields. Although many people observe optoelectronics in action in remote controls or compact disk (CD) players, most undergraduates do not get much exposure to optoelectronics in engineering curricula. This is particularly true at the freshman level. However, it is possible to introduce students to this area with a modest amount of expense.

An Optoelectronics Laboratory has been established and several laboratory experiments have been developed for undergraduates at Bucknell University with funding from an NSF Instrumentation and Laboratory Improvement (ILI) grant and the College of Engineering. One effort focused on an exercise for first year students. Another centered on adding a laboratory component to an upper level elective class in Optoelectronic Materials and Devices. The goals and level of these experiments varied and will be described in this paper. 


\section{BUCKNELL OPTOELECTRONICS LABORATORY}

The Bucknell Optoelectronics Laboratory has optical tables, a spectrometer, a white light source, a computer, an optical power meter, laser pointer, HeNe laser, the Newport Fiber Optics Kit, Optical Voice Link kit, and a laser diode controller. Various optical components, mounts, positioners, and lenses as well as electronic measuring equipment such as function generators, power supplies, multimeters, and oscilloscopes are also available to the students in lab. This facility was used for the elective class as well as senior design projects. Due to its small size, this room was not used for the freshmen lab. No special facilities are needed for the freshmen lab although one or two sinks are desirable.

\section{COURSES}

\section{EG100 Exploring Engineering}

Exploring Engineering (EG100) is a class of approximately 200 students, mostly first year engineers and some interested students from Arts and Sciences. ${ }^{1}$ In addition to lectures, students perform one lab from each of the five represented engineering disciplines: Chemical, Civil, Electrical, and Mechanical Engineering as well as Computer Science. As a senior design project in the Spring 1996 semester, Carrie Goldwein (EE'96) designed an optoelectronics laboratory for the EE lab portion of EG100. This project aimed to develop experiments that students would find interesting, teach them about optoelectronics, and give them a favorable impression of electrical engineering as something beyond circuits and wires. Because of the desire for the experiment to be inexpensive and still hands-on, the two hour lab period was divided into five modules. Students performed all five modules with each taking about fifteen minutes. An EE professor and at least one student assistant were present for the entire lab period. Equipment was kept to a minimum which permits the exercise to be transported to other schools. ${ }^{2}$ The cost for all stations is about $\$ 160$ including a laser pointer.

The modules investigate an optical voice link (OVL), total internal reflection (TIR), waveguiding (WG), different types of emitters (DTE), and different color LEDs (LED). Detailed instructions were provided as well as questions for the students. The OVL module illustrated how light and plastic optical fiber can be used to carry a signal from one point to another. Students were encouraged to experiment with speaking into the transmitter and hearing their voice on the receiver. TIR allowed students to observe the law of total internal reflection and use that to describe how a light beam travels in a fiber optic cable. WG showed students how a material such as water can be used to guide light, much like a fiber guides light in telecommunications. DTE had students compare a light bulb, a light emitting diode (LED), and a laser focusing on polarity, polarization, and the spatial extent of the beam. Students constructed a simple circuit to observe four LEDs in action in the LED module. They explored the differences between visible and infrared (IR) light and learned why different LEDs emit different colors. LEDs with clear packages were specifically chosen so the color of the package would not indicate the color of the emitted light. For more details on the development of this exercise, please consult the reference by Goldwein and Lord. ${ }^{3}$ The laboratory instructions including parts lists are available on the World Wide Web at http://www.eg.bucknell.edu/ ee378/eg100lab/design.html. 
This laboratory exercise was used as the EE lab in EG 100 Exploring Engineering for the Fall of 1996. Overall student response to the lab was positive. A questionnaire was given to the students at the end of the two hour lab period. $82 \%$ of the students rated the lab as very good or excellent overall while $77 \%$ found it interesting or very interesting. $95 \%$ of the students rated the level of difficulty as average or easier. Although we expected that most students would not associate optoelectronics with $\mathrm{EE}$, the responses to this questionnaire indicated that more students did $(55 \%)$ than did not $(45 \%)$ associate optoelectronics with EE before this lab. The Optical Voice Link (OVL) was selected as the "Favorite station" although it was not voted the easiest station. "Wave Guiding" (WG) was selected as the easiest and the second favorite station. The two stations voted most difficult, "Different Color LEDs" (LEDs) and "Different Types of Emitters" (DTE) were also the least favorite stations. Students were given the opportunity to make any additional comments on the questionnaire. Some of these comments indicate that we had achieved our goals. For example, "I thought the lab was fun, informative and very interesting. I think I'll look into electrical engineering as a possible major." and "This is the ideal EG100 lab. It is informative, to the point, and laid out nicely. It wasn't tedious or too indepth. This was perfect as an introduction to electrical engineering."

The departmental laboratories were frequently mentioned as a strength of the EG100 course on the end of the semester evaluations. The responses of students on the questionnaires at the end of the EE lab period were consistent with their responses on the end of the semester evaluations. The EE lab was rated very effective by more students (32\%) than any other lab. Another $44 \%$ rated the EE lab as effective. Its overall ranking was 4.0 out of a possible 5.0 making it one of the two most favorite labs of the students. The students in EG100 appreciated the upper class students who served as laboratory assistants as evidenced by the comment on the end of the semester evaluation that a strength of the course was "labs such as EE where T.A.s were accessible to talk to". Aside from the one masters student, none of the student assistants had taken a course in optoelectronics or performed this laboratory before the fall 1996 semester. Thus this lab does not require assistants who are highly trained in optoelectronics.

Some changes were made from the original design as the lab was implemented. While the two water stations (WG and TIR) went the fastest, DTE took the longest time so students were permitted to skip the question on polarization. A typical group included four students. However, when possible, students were broken into smaller groups to allow for more active participation. At one of the stations, the instructor showed students examples of glass optical fibers such as those used in telecommunications. This also provided an opportunity to explore various uses of Kevlar in addition to protecting optical fibers. In the LED module, a fair number of LEDs burned out when students did not connect a resistor in the circuit. A simple circuit diagram might help minimize this problem.

Students were graded on their responses to questions from instructors in lab as well as a brief memo focusing on what they learned which was due one week after the lab period. Some of these memos were excellent.

\section{EE 341 Optoelectronic Materials and Devices}

In the spring of 1996, a major improvement was made to the elective course, ${ }^{4}$ Optoelectronic Materials and Devices, with the addition of a laboratory component. Not only could students 
learn about lasers and fiber optics in lecture, they could also experiment with them in lab. Five new laboratory experiments were performed by the students as shown in Table 1. The first lab, $X$-ray Diffraction, was performed individually by the students. They learned how to determine the crystal lattice parameters of elemental semiconductors and alloys from X-ray diffraction data and to interpret precession photographs. X-ray diffraction is an important characterization tool for various optoelectronic materials especially semiconductors. Often, information on the lattice constant obtained from X-ray diffraction is used to determine an alloy's composition and thus its emission wavelength or wavelength detection range. This experiment was designed by Helen Kantes (EE '96) as part of her independent project in the spring of 1996. She conducted the lab for the students in the Optoelectronics Materials and Devices course including collecting student evaluations, developing a grading scheme, and initial grading.

In the remaining labs, students worked in groups of two. Each lab session included two groups and each experiment included two sections. Thus it took several lab periods for the entire class to perform the experiment. This was necessary due to the expense of the equipment in the Bucknell Optoelectronics Laboratory and the impracticality of having duplicates as well as the desire for students to be active participants in lab rather than passive onlookers.

TABLE 1: Experiments performed in Optoelectronic Materials and Devices in Spring 1996

\begin{tabular}{|l|l|}
\hline $\begin{array}{l}\text { Laboratory } \\
\text { Number }\end{array}$ & Laboratory Title \\
\hline 1 & X-ray Diffraction \\
\hline 2 & Sources of Light \\
\hline 3 & Detectors of Light \\
\hline 4 & Coupling Light into Multimode Fiber \\
\hline 5 & Creating Optical Communication Links \\
\hline
\end{tabular}

In the second lab, Sources of Light, students learned about different sources of light including white light sources, gas (Helium Neon or HeNe) and semiconductor (diode) lasers, and light emitting diodes (LEDs). Specifically, students focused on optical intensity versus wavelength and for the diodes, optical power versus input electrical current. In the third lab, Detectors of Light, students learned to characterize materials for detectors and compare photodetector device structures. Using transmission measurements, students analyzed the absorption properties of several samples including one of their own choosing that they brought to lab. Their samples ranged from "UV Blocking" sunglasses to a photographic negative. Several students particularly enjoyed the opportunity to bring their own samples. Students also compared the response times of different detectors including a phototransistor, photoconductor, and photodiode. ${ }^{5}$

The fourth and fifth laboratory experiments focused on fiber optics and were developed by Crystal Theesfeld (EE'96) ${ }^{6}$ as part of her senior design project. Students coupled light from a HeNe laser into an optical fiber in Coupling Light into Multimode Fiber using a microscope objective lens and state of the art fiber positioning mounts. Students calculated the loss of their system and explored ideas about fiber loss versus coupling loss for gas lasers and diode lasers. In Creating Optical Communication Links, students investigated digital modulation using a plastic fiber to send Morse code. Students also explored analog modulation in free space and optical fibers. Finally, students multiplexed two stereo signals: one using an external modulator and one using a directly modulated LED. For more details on the development of the fiber optic labs, please consult the reference by Theesfeld and Lord. ${ }^{7}$ The laboratory handouts and other 
documentation is also available on the World Wide Web at http://www.eg.bucknell.edu/ ee378/341/index2.html.

Student feedback on all of the laboratory experiments was collected and considered vital to improving the labs. On the end of the 1996 spring semester evaluations, all ten students in the class rated the usefulness of the laboratory experiments as very good or excellent. When asked which laboratory was their favorite for the course, each lab received at least one vote. Lab 5 Creating Optical Communication Links was the class favorite with $40 \%$ choosing it as exemplified by the comments "it was pretty cool to be able to transmit sounds and music that was being modulated by light. It was cool to be able to hear the music from both tapes in the single fiber after they were coupled into the same fiber by the grin rod lens" and "it was incredible how music traveled as light through an optical fiber". Lab 4 and Lab 1 each received two votes or $20 \%$. One student remarked that "I never pictured that stuff X-ray Diffraction[ to have anything to do with optoelectronics." One student chose Lab 2 calling it the "most straight forward" and another chose Lab 3 because it "illustrated concepts clearly, I learned a lot." Three students chose Lab 1 as their least favorite laboratory with their reasons including "This lab wasn't too clear. It involved a little more chemistry which is a weak subject for me." and "I don't think I left with an understanding of the main concepts" and "I'm still not sure what it had to do with optoelectronics." Two students chose Lab 4 citing unclear objectives and frustration in aligning the beam. Two students chose Lab 5 because they could not get it to work or felt it was "too much material for one lab write-up too vague." Three students did not have a least favorite laboratory. Certainly an inherent risk of fiber optic laboratory experiments such as Labs 4 and 5 is that there are no guarantees that students will be successful. Efforts were made to minimize the amount of alignment required and the instructor and student laboratory assistant provided assistance but there is no exact recipe for coupling light into fibers. It is useful if painful for students to learn that some tasks demand patience and luck.

\section{SUMMARY}

ILI funding has been used in the development of an Optoelectronics Laboratory at Bucknell University. This project also included developing a laboratory exercise for first year students as well as a laboratory sequence for an elective course on Optoelectronic Materials and Devices. The first year student exercise and two labs for the elective course were developed as senior design projects. Both senior design projects served well as two tiered learning experiences. The senior design students learned about the process of designing a lab and the students performing the laboratories learning about optoelectronics. Involving undergraduates in development of laboratories can be a valuable pedagogical technique.

\section{ACKNOWLEDGMENTS}

Partial support for this work was provided by NSF's Division of Undergraduate Education through Instrumentation and Laboratory Improvement (ILI) Grant DUE \#9552260.

The author gratefully acknowledges the contributions of Carrie Goldwein (EE'96) and Crystal Theesfeld (EE'96) through their senior design projects. The patience and suggestions of the students in EG100 F96 and EE 341 S96 were invaluable for the success of this endeavor. The 
support and encouragement of Maurice Aburdene and Victor Chang are also sincerely appreciated.

\section{REFERENCES}

1. For example, see: Aburdene, M. F., R. G. McGinnis and D. J. Cartwright, "Exploring Engineering," Frontiers in Education Conference, 1990, Vienna, Austria. and Schuster, D. S., T. B. Cunningham, and S. A. Richardson, "Exploring Engineering -- The Five Year Evolution of an Interdisciplinary Engineering Course," World Conference on Engineering Education, 1995, Minneapolis, Minnesota.

2. Professor Emily Allen at San Jose State University used this laboratory in MatE 153 "Electronic, Optical, and Magnetic Properties of Materials" in November 1996. Prof. Allen says "It also demonstrates how important the Web is for dissemination, because it made it so easy that I actually did the experiment in class (with sections totaling 60 students) within two weeks of hearing Carrie's talk [at FIE96]!"

3. Carrie H. Goldwein and Susan M. Lord, "Optoelectronics Laboratory For First Year Students," Session 7c2, Proceedings of the 1996 Frontiers in Education Conference, Salt Lake City, Utah, November 1996. Available at http://www.caeme.elen.utah.edu/fie

4. Susan M. Lord, "A Multidisciplinary Elective on Optoelectronics," Session 7c2, Proceedings of the 1995 Frontiers in Education Conference, Atlanta, Georgia, November 1995.

5. From Experiment IV of the Fiber Optic Lab Manual by Terry G. White of Industrial Fiber Optics.

6. Crystal now works for Corning, the premier manufacturer of optical fibers. A highlight of this project was her comment "If someone had come to me a year ago and said that I would really like something in EE, I would have said they were crazy. But I really like this stuff. I don't mind spending time in the lab to get it to work."

7. Crystal J. Theesfeld and Susan M. Lord, "Designing Optoelectronic Laboratories: A Unique Senior Design Opportunity," Session 7c2, Proceedings of the 1996 Frontiers in Education Conference, Salt Lake City, Utah, November 1996. Available at http://www.caeme.elen.utah.edu/fie/

\section{SUSAN M. LORD}

Susan M. Lord received a B. S. in Electrical Engineering \& Materials Science from Cornell University and the M.S. and Ph.D. in Electrical Engineering from Stanford University. Dr. Lord has been an Assistant Professor of Electrical Engineering at Bucknell University since August 1993 and will join the faculty of the University of San Diego in September 1997. Her research interests include optoelectronic materials and device characterization and diode lidar. 